\title{
ON POWERS OF ELEMENTS IN $S$-GROUPS
}

\section{SEYMOUR LIPSCHUTZ ${ }^{1}$}

1. Introduction. Greendlinger proved that in $S$-groups [2] (see §2) each conjugate class contains at most a finite number of the powers of a generator. In this paper we extend this result. We prove that each power of a generator is in a different conjugate class. We also exhibit other elements with this same property.

The methods used in this paper, which include the use of "cyclic" words, were developed in a paper by Dehn [1]. These methods were used by Greendlinger to solve the word problem [4] for $S$-groups, the transformation problem for a smaller class of groups [3] and, lately, the transformation problem for $S$-groups. ${ }^{2}$ The author also used these methods in a previous paper [5] on $S$-groups.

2. Notations and definitions. Let a group $G$ be generated by $a, b, \cdots$ with defining relations $R_{1}=1, R_{2}=1, \cdots$. We denote this group by

$$
G=\left\langle a, b, \cdots ; R_{1}, R_{2}, \cdots\right\rangle,
$$

where we call the $R_{\boldsymbol{i}}$ relators. We assume all relators are cyclicly reduced. Also, we include in the set of relators in (9) all cyclic permutations of the symbols of $R_{l}^{ \pm 1}$. If $R \equiv a_{1} a_{2} \cdots a_{n}, R^{\prime} \equiv b_{1} b_{2} \cdots b_{q}$, and $a_{n+1-i} \approx b_{i}^{-1}$ for $i=1,2, \cdots, p$ but not for $i=p+1$, then we say that $R$ reacts with $p / q$ of $R^{\prime}$, or that $R$ absorbs $B \equiv b_{1} b_{2} \cdots b_{p}$.

Definition. A group $G$ is an $S$-group (less than a sixth group) if, in the presentation (1), any relator $R$ reacts with less than $1 / 6$ of any other relator $R^{\prime}$-unless $R \equiv R^{\prime-1}$.

We use the following notation:

$\bar{W}$ for $W^{-1}$

$l(W)$ for the length of $W$

$R_{i}$ for a particular relator

$R$ for any relator

$W^{*}$ is any cyclic permutation of the word $W$

$A=B$ means $A$ and $B$ are the same element of the group $G$

$A \approx B$ means $A$ and $B$ are freely equal

$A \equiv B$ means $A$ is identical to the word $B$

Received by the editors March 8, 1961.

1 Work on this paper was sponsored by a grant from the National Science Foundation, NSF-G9659.

2 Unpublished. But proof is indicated in [3]. 
Capital letters will always denote (unless otherwise stated or implied) freely reduced words in which no subword is greater than $3 / 6$ of any relator. (Obviously, by a constructive process, any word can be put in this form.) Lower case letters will denote generators.

A word $W$ contains the set of words $\left\{V_{1}, V_{2}, \cdots, V_{n}\right\}$, written $W \supset\left\{V_{1}, \cdots, V_{n}\right\}$ or $\left\{V_{1}, \cdots, V_{n}\right\} \subset W$, if there exist words $S_{0}, S_{1}, \cdots, S_{n}$ such that $W \equiv S_{0} V_{i_{1}} S_{1} \cdots V_{i_{n}} S_{n}$. We naturally call the $V_{i}$ subwords of $W$.

A word $W$ cyclicly contains the set of words $\left\{V_{1}, \cdots, V_{n}\right\}$ if there exists a cyclic permutation $W^{*}$ of $W$ such that $W^{*} \supset\left\{V_{1}, \cdots, V_{n}\right\}$. (Note: This is equivalent to "bending $W$ into a circle"-where we write down its end in front of its beginning - and obtain the "cyclic" word corresponding to $W$.)

A word $W$ is greater than $p / q$ of a word $V$, written $W>p / q V$, if $W \subset V$ and $l(W)>p / q l(V)$. (Likewise for greater than or equal to.)

A word $W$ is less than $p / q$ of a word $V$, written $W<p / q V$, if $l(S)<p / q l(V)$ for every $S \subset W^{*}$ and $S \subset V$, that is, if every cyclicly contained word is not greater than or equal to $p / q$ of $V$. (Likewise for less than or equal to.)

A freely reduced word $W \equiv a_{1} a_{2} \cdots a_{n}$ is cyclicly reduced if $W$ does not react with itself, that is, if $a_{n}$ not $\approx \bar{a}_{1}$.

3. Preliminary lemmas. Throughout this paper we will assume that our group $G$ is an $S$-group. We also assume various trivial consequences of our definitions, e.g., $A<1 / 6 R, B<1 / 6 R$ implies $A B<2 / 6 R$. We state without proof the following lemma by Greendlinger. (See $[3 ; 4]$.)

GREENDLINGER'S LEMMA. If a cyclicly reduced word $W$ represents the identity element in an $S$-group then there is a cyclic permutation $W^{*}$ of $W$ such that one of the following is true:

(a) $W^{*}$ is a relator,

(b) $W^{*} \supset\left\{S_{1}, S_{2}\right\}$ where $S_{i}>5 / 6 R_{i}$,

(c) $W^{*} \supset\left\{S_{1}, S_{2}, S_{3}\right\}$ where $S_{i}>4 / 6 R_{i}$,

(d) $W^{*} \supset\left\{S_{1}, S_{2}, T_{3}, T_{4}\right\}$ where $S_{i}>4 / 6 R_{i}, T_{i}>3 / 6 R_{i}$,

(e) $W^{*} \supset\left\{S_{1}, S_{2}, S_{3}, S_{4}, S_{5}\right\}$ where $S_{i}>3 / 6 R_{i}$.

(The reader can easily see how Greendlinger's Lemma gives a solution to the word problem for $S$-groups.)

We first prove the following simple lemmas.

LEMma 1. If $R \equiv A V B \bar{V}$ then $V<1 / 6 R$.

Proof. $V$ will be absorbed when $R$ reacts with $R^{\prime} \equiv V B \bar{V} A$.

Lemma 2. If $V a \subset R$ where $V>1 / 6 R$ then $b \bar{V} \nsubseteq R^{\prime}$ unless $a \approx b$. 
Proof. $V>1 / 6 R$ is absorbed when $R^{\prime} \equiv S^{\prime} b \bar{V}$ reacts with $R \equiv V a S$. Thus $R^{\prime} \approx \bar{R}$ and $a \approx \bar{b}$.

LEMMA 3. If we freely reduce $W T \bar{W}$ we get $V T^{*} \bar{V}$ where $W$ begins with $V$ and $T^{*}$ is a cyclic permutation of $T$.

Proof. If $W \equiv V \bar{A}$ and $T \equiv A B$ then

$$
W T \bar{W} \equiv V A A B A \bar{V} \approx V B A \bar{V} \equiv V T^{*} V .
$$

LEMMA 4. If $l(R) \leqq 6$ then the symbols of $R$ appear in no other relators except the cyclic permutations of $R^{ \pm 1}$. Hence

$$
G=G^{\prime} * F,
$$

the free product of groups $G^{\prime}$ and $F$, where $G^{\prime}$ is also an $S$-group and $F$ is an $S$-group with only the relators $R^{ \pm 1}$ and their cyclic permutationsa group with one defining relation.

Proof. If $R \equiv a X$ then $R^{\prime} \equiv Y \bar{a}$ must be $R^{-1}$ since $R^{\prime}$ absorbs $a \geqq 1 / 6 R$.

LEмma 5. If $a \subset R$ where $l(R) \leqq 3$ then $R \equiv a^{n}$ or the powers of a are in different conjugate classes.

Proof. $R$ must be of the form

$$
a, a^{2}, a^{3}, a b \text {, or } a b c .
$$

(We cannot have $R \equiv a^{2} b$ else $R^{\prime} \equiv \bar{a} \bar{b} \bar{a}$ would absorb $1 / 3$ of $R$.) By Lemma $4, a$ is in a free factor $F$ of $G$ with only the defining relation $R$. In the first three cases $R \equiv a^{n}$. In the case $R \equiv a b$ (or $R \equiv a b c$ ), $F$ is a free group on one (or two) generator. Therefore, by known theorems on free groups and free products, the powers of $a$ are in different conjugate classes.

4. Main Lemma. Let $A$ and $B$ be cyclicly reduced and let $A, B$ $<2 / 6 R$. If $A$ is conjugate to $B$ in $G$ then $A \equiv B^{*}$, a cyclic permutation of $B$.

Proof. If $A$ is conjugate to $B$ then there exists a word $S$ in $G$ such that

\section{$\bar{A} S B \bar{S}$}

represents the identity element. If we cyclicly reduce (2) we have three possibilities: Case I, (2) reduces to the empty word. Case II, $S$ is absorbed so, by Lemma $3,(2)$ reduces to $A^{\prime} B^{\prime}$ where $A^{\prime} \subset A^{*}$ and $B^{\prime} \subset B^{*}$. Case III, (2) reduces to

$$
W T V \bar{T}
$$


where, by Lemma $3, W \equiv \bar{A}^{*}, V \equiv B^{*}$ and $T \subset S$. (Note that $W, V$ $<2 / 6 R$.)

Since $A, B<2 / 6 R$ implies $A^{\prime} B^{\prime}<4 / 6 R$, we can conclude that $A^{\prime} B^{\prime}$ cannot satisfy Greendlinger's Lemma. Thus Case II cannot happen. Since Case I implies our conclusion, that is, $A \equiv B^{*}$, the proof of our lemma now reduces to proving that (3) also cannot satisfy any of the cases of Greendlinger's Lemma. Note: as different $S$ in (2) give rise to different words of the form (3), we further assume, without loss in generality, that (3) is of minimal length in the class of words of the form of (3).

Case (a). If (3) is a relator then, by Lemma $1, T<1 / 6 R$. This implies that $W T V \bar{T}<6 / 6 R$ since $W, V<2 / 6 R$. Thus (3) is not a relator.

Cases (c), (d) and (e). We show that (3) cannot cyclicly contain $S_{1}$, $S_{2}, S_{3}$ where $S_{i}>3 / 6 R_{i}$. We write (3) around a square as in Figure 1. We see, as in Figure 1, that each of the $S_{i}$, being greater than $3 / 6$

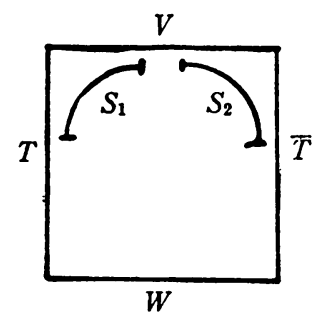

Figure 1

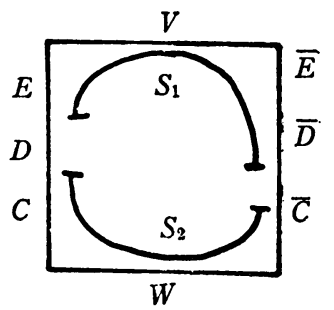

Figure 2

of $R_{i}$, must cross one of the vertices of the square; that is, cannot be contained in $T, V, \bar{T}$ or $W$ alone. Also, we see that two of the $S_{i}$, say $S_{1}$ and $S_{2}$, must cross the upper corners (or the lower corners) of the square.

As $V \equiv v_{1} v_{2} \cdots v_{n}<2 / 6 R, T$ must end in a subword $X$ where $X v_{1} \subset S_{1}, v_{n} \bar{X} \subset S_{2}$ and where $X>1 / 6 R_{1}$ or $R_{2}$. As $V$ is cyclicly reduced, this violates Lemma 2. Thus (3) cannot satisfy Cases (c), (d) or (e) of Greendlinger's Lemma.

Case (b). Lastly, suppose (3) cyclicly contains some $S_{1}, S_{2}$ where $S_{i}>5 / 6 R_{i}$. As $V, W<2 / 6 R$, each $S_{i}$ cannot be contained in $T V$, $V \bar{T}, \bar{T} W$ or $W T$ alone. Therefore $S_{1}$ and $S_{2}$ must cross the upper and the lower vertices of the square as in Figure 2, or $S_{1}$ and $S_{2}$ must cross the left and the right vertices of the square as in Figure 3.

Suppose, as in Figure 2, that $V \subset S_{1}$, say $S_{1} \equiv E V \bar{E} \bar{D}, T \equiv C D E$, $R_{1} \equiv E V \bar{E} \bar{D} X$. By Lemma $1, E<1 / 6 R_{1}$. Therefore $D>1 / 6 R_{1}$. But $S_{1}>5 / 6 R_{1}$ implies $X<1 / 6 R_{1}$. Thus $l(X)<l(D)$. 


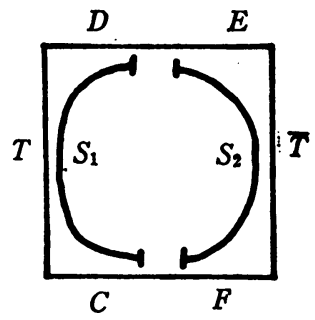

FIGURE 3

Since $R_{1} \equiv E V \bar{E} \bar{D} X$, we note that $D=X E V \bar{E}$ and $\bar{X}=E V \bar{E} \bar{D}$. Substituting in (3) we have

$$
W T V \bar{T} \equiv W C D E V \bar{E} \overline{D C}=W C X E V \bar{E} \bar{X} \bar{C} \equiv W T^{\prime} V \bar{T}^{\prime},
$$

which is of the form of (3) if $T^{\prime} \not \equiv 1$. (We let $T^{\prime} \equiv C X E$.) But $l(X)$ $<l(D)$ implies $l\left(T^{\prime}\right)<l(T)$, which then implies that $l\left(W T^{\prime} V \bar{T}^{\prime}\right)$ $<l(W T V \bar{T})$. This last statement contradicts the original assumption that (3) was of minimal length in the class of words of the form of (3). We can also exclude the possibility that $T^{\prime} \equiv 1$ since $W V<4 / 6 R$ cannot satisfy Greendlinger's Lemma. Thus $V$ cannot be contained in $S_{1}$, as in Figure 2.

Now suppose, as in Figure 3 , that $T \subset S_{1}$ and $\bar{T} \subset S_{2}$, say $S_{1} \equiv C T D, S_{2} \equiv E \bar{T} F$ where $C, D, E, F$ are not empty. But $W, V$ $<2 / 6 R$ implies $C, D, E, F<2 / 6 R$, which implies that $T>1 / 6 R$. By the same argument as in Case (c), we again have a contradiction.

Thus (3) does not satisfy Greendlinger's Lemma, so our lemma is true.

THEOREM 1. Every power of a generator a in an S-group is in a different conjugate class unless there is a relator $R \equiv a^{n}$.

Proof. In view of Lemma 5, we need only consider relators $R$ for which $l(R)>3$. We show that, for any $s, a^{s}<2 / 6 R$-unless $R \equiv a^{n}$. For $s= \pm 1$, the statement is true since $l(R)>3$. Now suppose $s \neq \pm 1$, say $R \equiv A a^{s}$ where $A \not \equiv a^{r}$. Then at least $a^{s-1}$ is absorbed when $R$ reacts with $R^{\prime} \equiv a^{1-s} \bar{A} \bar{a}$. Thus $a^{s-1}<1 / 6 R$. Thus $a^{s}<2 / 6 R$.

But $a^{*} \neq a^{\imath *}$ if $s \neq t$. Therefore the proof of our theorem follows directly from our Main Lemma and Lemma 5.

We let the reader prove, in a manner parallel to the proof of the Main Lemma, the following special case:

'THEOREM $1^{\prime}$. If there is a relator $R \equiv a^{n}$ in an $S$-group, then each power $i$ of $a, i=1,2, \cdots, n$, is in a different conjugate class. 
THEOREM 2. If $W$ is cyclicly reduced and $W<1 / 6 R$, then every power of $W$ is in a different conjugate class unless there is a word $C$ such that $W \equiv C^{n}$ and $R \equiv C^{m}$ for some $m$ and $n$.

Proof. The proof follows in the same manner as in Theorem 1.

5. Application. It is long known [2] that groups with presentations

$$
G=\left\langle a, b ; a^{r}, b^{*},(a b)^{t}\right\rangle \quad(r, s, t=1,2, \cdots),
$$

are a subclass of the discontinuous Fuchsian Groups. The reader can easily verify that (4) are $S$-groups if $r, s, t>6$; and that $W \equiv a b^{-1}$ $<1 / 6 R$. Thus all powers of $W$ are in different conjugate classes. Thus we have a purely algebraic proof that the groups (4) are infinite. In fact, we have also shown that they have an infinite number of conjugate classes.

\section{BIBLIOGRAPHY}

1. M. Dehn, Transformation der Kurven auf zweiseitigen Flaechen, Math. Ann. 72 (1912), 413-421.

2. R. Fricke and F. Klein, Vorlesungen ilber die Theorie der automorphen Funktionen, Vol. 1, B. G. Teubner, Leipzig, 1897.

3. M. Greendlinger, On Dehn's algorithms for the conjugacy and the word problems, with applications, Comm. Pure Appl. Math. 13 (1960), 641-677.

4. - Dehn's algorithm for the word problem, Comm. Pure Appl. Math. 13 (1960), 67-83.

5. S. Lipschutz, Elements in S-groups with trivial centralizers, Comm. Pure Appl. Math. 13 (1960), 679-683.

New York University 